\title{
In situ precipitation of gold nanoparticles onto glass for potential architectural applications
}

\author{
Xiaoda Xu ${ }^{a}$, M. Stevens ${ }^{b}$ and M.B. Cortie ${ }^{* a}$ \\ ${ }^{a}$ Institute for Nanoscale Technology, University of Technology Sydney, Australia \\ ${ }^{\mathrm{b}}$ Department of Chemistry, Materials and Forensic Science, University of Technology Sydney, \\ Australia
}

Key words: gold nanoparticles, glass, infrared reflective coating

PO Box 123, Broadway, NSW 2007, Australia

Tel +61 29514 2208, Fax +61295147553

Email: Michael.cortie@uts.edu.au 


\begin{abstract}
The optical properties of in situ deposited gold nano-particle coatings are investigated for potential application in architectural glass. It is found that the optical properties of the coating can be controlled by the $\mathrm{pH}$ of the deposition solution. At a $\mathrm{pH}$ of 5.1 the color of the coatings develops from pink, through violet, to blue in transmission. This is due to a plasmon resonance peak at 520 $\mathrm{nm}$ from isolated particles, and one at about $700 \mathrm{~nm}$ due to near-field dipole interactions, with an intermediate zone of coexistence of the two, which produces the violet color. However, the two peaks do not coexist in the spectra of coatings produced at $\mathrm{pH} 8.0$ or at $\mathrm{pH} 10.0$, with the peak due to the $520 \mathrm{~nm}$ resonance being swamped by the development of the resonance due to particleparticle interactions. In all cases the $700 \mathrm{~nm}$ peak could be broadened and red-shifted by increasing the deposition time. The reasons for these differences are explored, and are shown to be attributable to the smaller, more aggregated morphology of nano-particles precipitated at the higher pHs. The wavelength of maximum plasmon resonance is examined as a function of the volume fraction of nanoparticles. Significant deviations from the well-known Genzel-Martin analytical model are observed. The reasons for deviation of the model are discussed. Finally, it is shown how coatings that are blue or blue-gray in transmission can be obtained by exploiting this deviation. Such coatings may be more suitable for architectural application than the conventional pink-hued coatings obtained with colloidal gold nanoparticles.
\end{abstract}




\section{Xiaoda Xu, M. Stevens and M.B. Cortie *}

Chem. Mater.

In situ precipitation of gold nanoparticles onto glass for potential architectural applications
Coatings of similar average density and similar nominal particle size, but different patterns of aggregation, can produce quite different optical transmission spectra, (a) shows substantially isolated particles of $60 \mathrm{~nm}$ diameter, and the corresponding spectrum shows a peak at $520 \mathrm{~nm}$, whereas (b) is aggregated from much smaller particles and has a broad absorption peak at approximately $655 \mathrm{~nm}$.

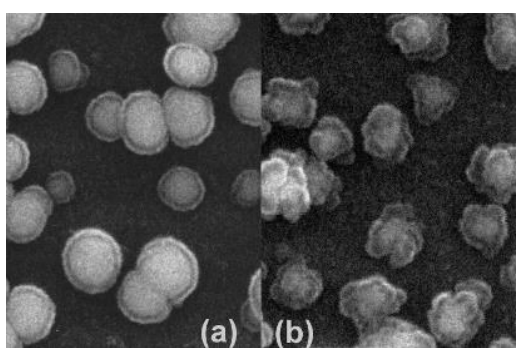




\section{Introduction}

Ordinary window glass of $4 \mathrm{~mm}$ thickness transmits about $90 \%$ of incident visible and near-infra red light. ${ }^{1}$ Solar radiation, as received on Earth, ${ }^{2}$ is also substantially concentrated on this region of the spectrum (Figure 1). This implies that a considerable amount of energy can be transmitted into a building interior through windows that are directly illuminated by sunlight. For example, at $32^{\circ}$ latitude an un-shaded east or west-facing window can receive up $700 \mathrm{~W} / \mathrm{m}^{2}$ of solar energy during daylight hours. ${ }^{1}$ This can cause a heat load of tens of kilowatts into a residence or office space. This energy, while welcome in winter, is generally not desired in summer. Efforts to remove it by air conditioning contribute significantly to national energy consumption, and hence greenhouse gas emissions, in many warmer regions of the developed world ${ }^{3,4}$.

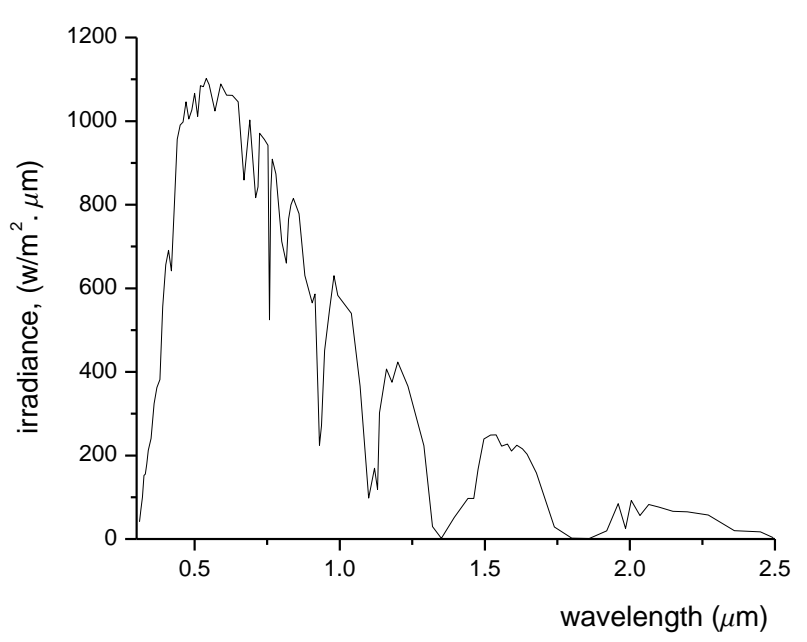

Fig. 1 Direct normal solar spectral irradiance at air mass 1.5 for a $37^{\circ}$ tilted surface ${ }^{2}$

A possible solution is to modify windows so that they attenuate incoming solar radiation. Up to $50 \%$ of the incoming visible spectrum can be removed while still retaining a degree of optical transparency that is acceptable to most consumers. ${ }^{1}$ 
In the present paper we will show how a coating of gold nano-particles on glass can serve for solar screening applications. However, to place the work in context we will first briefly mention what the existing coating technologies for glass are, and why we believe a coating of gold nano-particles has some potential use for this application.

'Pyrolytic' coatings of tin and/or indium oxides on glass have been used since the 1950s, and are applied by subjecting glass at approximately $600^{\circ} \mathrm{C}$ to a spray or vapour from which the transparent oxide is deposited by pyrolysis. ${ }^{1}$ The coatings reflect a proportion of the infra-red (IR) radiation falling on the glass while transmitting most of the visible light. Pyrolytic coatings are durable, however the disadvantage of the process is the requirement that it can only be applied at the time of manufacture of the glass. Another scheme is to deposit partially reflective films of Au, Ag, TiN, $\mathrm{ZrN}, \mathrm{Cr}_{\mathrm{X}} \mathrm{N}, \mathrm{Si}_{3} \mathrm{~N}_{4}, \mathrm{SnO}_{2}$ or $\mathrm{In}_{2} \mathrm{O}_{3}$ onto glass by vacuum sputtering ${ }^{1,5}$. In this case the glass is at ambient temperature during the process. These films are usually sandwiched between layers of transparent dielectrics of high refractive index, such as $\mathrm{ZnO}, \mathrm{Nb}_{2} \mathrm{O}_{5}, \mathrm{Bi}_{2} \mathrm{O}_{3}$, or $\mathrm{TiO}_{2}{ }^{5,6}$ The industrial-scale production of large areas of such coated glasses, using magnetron sputtering, began in the early 1980s and is growing steadily. ${ }^{7}$ Unfortunately, the high cost of applying these coatings (e.g. $\sim \$ 10$ per $\mathrm{m}^{2}$ ) is a dominating aspect of the economics, even though the actual cost of the raw materials used (e.g. $\$ 1$ or less per $\left.\mathrm{m}^{2}\right)$ is hardly significant.

The alternative strategy of absorbing incident IR radiation (rather than reflecting it) has also attracted interest. ${ }^{8}$ This is achieved by dispersing a nano-particulate material such as $\mathrm{FeO}_{\mathrm{x}}$ or $\mathrm{LaB}_{6}$ with strong IR absorptive characteristics either into the glass itself ('tinted' glass), or into a polymer film applied onto the glass ('plastic-filmed glass'). ${ }^{8,9}$ The advantage of a plastic film is that it can be retro-fitted to a glass pane at any time. Of course, IR-reflective plastic films are also available. A significant drawback to IR absorbing systems is that they shed about $50 \%$ of their absorbed energy into the interior environment. ${ }^{1}$ 
It is our contention that the diverse disadvantages of the existing technologies have created a niche in the market for a new solar screening coating, provided that it is cheap to apply and has acceptable spectral properties.

Gold nanoparticles have the interesting property of exhibiting a plasmon resonance with light at a nominal wavelength of around $520 \mathrm{~nm}$. This phenomenon has been known for about 150 years and has been much studied. In transmission, this absorption removes the green component of white light and causes the substance containing the nano-particles to exhibit a pink-red colour. The wavelength at which resonance occurs with isolated gold nano-particles is a comparatively weak function of particle diameter, ${ }^{10,11}$ varying from about 510 to $580 \mathrm{~nm}$ as particle size is increased from 20 to 100 nm. ${ }^{11,12}$ However, a decreased spacing and/or aggregation of the particles, which would usually accompany an increase in particle size and volume fraction, is associated with the development of a second resonance, starting at $680 \mathrm{~nm}$, and which red-shifts strongly into the near infrared as the particles pack more closely together. ${ }^{12,13}$ Coating thickness and the dielectric properties of the surrounding matrix (if any), ${ }^{13}$ the particle morphology, ${ }^{11}$ and the regularity of spacing ${ }^{14}$ also influence color. However, such interest as there has been in depositing gold nanoparticles onto glass has been ostensibly directed at developing photonic materials, ${ }^{13,15}$ or towards enhancing the Raman response of gold in order to provide a more sensitive analytical technique for organic molecules. 16,17,18. In these latter cases gold nanoparticles were prepared separately and then deposited onto a glass substrate. Special treatments of the glass and/or nanoparticles surfaces were required to ensure adhesion of the particles onto the glass. ${ }^{16}$

The idea of an aqueous process to precipitate transparent gold coatings onto glass for architectural applications is not new, and dates from at least the patent of Miller in $1977 .{ }^{19}$ However, many of the early methods required the use of currently unattractive chemicals such as cyanides, and/or soluble salts of lead, mercury or cadmium. For whatever reason, these processes seem not to have been 
widely exploited. Our interest has been to explore whether, with the benefit of current knowledge of nanotechnology, a coating of gold nanoparticles could be developed that was simple to apply, useful for solar glazing, and which did not require the use of hazardous raw materials.

It is necessary that we first address a possible objection to such coatings for solar glazing based on the cost of the gold that they contain. A layer of gold nanoparticles equivalent in volume to a continuous coating of $10 \mathrm{~nm}$ of $\mathrm{Au}$ (a useful thickness for solar glazing) would contain US\$1.86 $/ \mathrm{m}^{2}$ of gold (calculated at US\$300/troy ounce). This is much less than the total cost (capital plus operating) of vacuum coating glass with any material. It is also less than $1 \%$ of the retail cost of retrofitted film-based schemes. Therefore, we believe that such a product could be viable provided that it was effective, cheaply applied, and possessed a hue that was acceptable to consumers.

\section{Experimental}

The adhesion of colloidal gold nano-particles onto ordinary glass is poor ${ }^{16}$ and merely dipping a glass surface into such a solution will not result in significant adhesion of gold nanoparticles. Therefore specialised techniques are required. In recent work the approach taken has been to functionalise the surface of the gold nanoparticles, or the glass, or both, so that previously prepared nanoparticles adhere onto the glass. ${ }^{13,16}$ In general, relatively complex reagents, such as aminoalkylalkoxysilanes ${ }^{13,16,20}$ are required to achieve this.

In our work we have taken a different approach, inspired by the now-lapsed patent literature on electroless deposition of gold. In this approach, the reduction of $\mathrm{Au}^{3+}$ to form nanoparticles is arranged to occur heterogeneously on the surface of the glass, rather than homogeneously in the solution. The basic concept may be found in the patents of Levy ${ }^{21}$ for decorative, non-transparent coatings, from which we have drawn the idea of using ethylene diamine as a complexing agent, of 
Miller, ${ }^{19}$ from whom we have taken the method of using a stannous chloride pre-treatment, of Franz and Vanek, ${ }^{22}$ from whom we have taken the clue of buffering the solution to control $\mathrm{pH}$, and of Luce, ${ }^{23}$ who used $\mathrm{HAuCl}_{4}$ as a source of $\mathrm{Au}^{3+}$ and hydrazine as the reducing agent.

The ethylene diamine, hydrazine, and sodium hydroxide were obtained from Aldrich, while $\mathrm{HCl}$, $\mathrm{H}_{2} \mathrm{SO}_{4}, \mathrm{HNO}_{3}$, potassium hydrogen phthalate, $\mathrm{KCl}, \mathrm{SnCl}_{2} \cdot 6 \mathrm{H}_{2} \mathrm{O}$, and gold were sourced from diverse suppliers. All chemicals were used as-received. Generic soda-lime glass microscope slides were obtained from Livingstone. All $\mathrm{H}_{2} \mathrm{O}$ used was purified by double-distillation.

$\mathrm{HAuCl}_{4}$ was prepared by dissolving pure gold in aqua regia $\left(\mathrm{HCl}: \mathrm{HNO}_{3} 3: 1 \mathrm{~V} / \mathrm{V}\right)$. The method may be found elsewhere. ${ }^{24} \mathrm{~A}$ brief description of the procedure is as follows: $9.85 \mathrm{~g}$ pure gold was dissolved in $80 \mathrm{ml}$ of aqua regia and the solvent was evaporated under aspirator vacuum on a water bath. For removal of nitric acid, the product was strongly heated twice with concentrated $\mathrm{HCl}$ in the same manner; the excess hydrochloric acid is separated by concentrating the remaining solution to around $15 \mathrm{ml}$. Finally the solution was diluted to $500 \mathrm{ml}$ to get $0.10 \mathrm{M} \mathrm{HAuCl}_{4}$ solution. It is accepted that some residual $\mathrm{HCl}$ content will have been present in the solution.

We pre-treated the glass surface by immersing for one hour in $5 \% \mathrm{NaOH} /$ methanol solution and then 2 minutes in $0.1 \% \mathrm{SnCl}_{2}$ in water. The slide was rinsed with pure water before deposition of the gold from a solution of $0.0004 \mathrm{M} \mathrm{HAuCl}_{4}, 0.015 \mathrm{M} \mathrm{N}_{2} \mathrm{H}_{4}$ and $0.03 \mathrm{M}$ ethylene diamine. This solution was buffered to $\mathrm{pH}$ values of $5.1,8.0$ or 10.0 . The $\mathrm{pH} 5.1$ buffer solution was prepared by mixing $100 \mathrm{ml}$ of $0.1 \mathrm{M}$ potassium hydrogen phthalate and $51 \mathrm{ml}$ of $0.1 \mathrm{M} \mathrm{NaOH}$. The $\mathrm{pH} 8.0$ buffer was prepared by mixing $100 \mathrm{ml} 0.025 \mathrm{M} \mathrm{Na}_{2} \mathrm{~B}_{4} \mathrm{O}_{7} .10 \mathrm{H}_{2} \mathrm{O}$ (borax) with $41 \mathrm{ml} 0.1 \mathrm{M} \mathrm{HCl}$. The pH 10.0 buffer solution was prepared by mixing $100 \mathrm{ml} 0.05 \mathrm{M} \mathrm{NaHCO}_{3}$ with $21.4 \mathrm{ml} 0.1 \mathrm{M}$ $\mathrm{NaOH}$. It was confirmed that the $\mathrm{pH}$ of these solutions stayed constant during the deposition.

The coating solution must be made up immediately prior to use, and has a short lifetime of minutes to hour, depending on $\mathrm{pH}$. The published Pourbaix diagram for $\left[\mathrm{AuCl}_{4}\right]^{-}$shows that the gold 
should form soluble complexes in the buffered environments provided that the $\left[\mathrm{Cl}^{-}\right]$concentration was greater than about $10^{-2} \mathrm{M} .{ }^{25}$ The soluble species in the $\mathrm{pH} 5.1$ environment was likely to have been $\left[\mathrm{AuCl}_{3} \mathrm{OH}\right]^{-}$or $\left[\mathrm{AuCl}_{2}(\mathrm{OH})_{2}\right]^{-}$, whereas that in the alkaline buffer was likely to have been $\left[\mathrm{AuCl}(\mathrm{OH})_{3}\right]^{-}$

A Cary 5E UV/Vis/IR spectrophotometer, working in the range of $170 \mathrm{~nm}$ to $4300 \mathrm{~nm}$ and with a resolution of $0.5 \mathrm{~nm}$ was used to inspect the visible-IR transmission spectrum of the samples. The scanning rate was $5 \mathrm{~nm} / \mathrm{s}$ with spectral bandwidth (SBW) of $2 \mathrm{~nm}$.

The coverage and nature of the coating of gold nanoparticles was assessed using a combination of X-ray photoelectron spectroscopy (XPS), scanning electron microscopy (SEM) and atomic force microscopy (AFM). XPS spectra of the $\mathrm{Au}$ nanoparticles were recorded using $\mathrm{Al} \mathrm{K}_{\alpha}$ radiation $(1486.6 \mathrm{eV})$ at $20 \mathrm{eV}$. The sample was placed in an ultra high vacuum chamber at $10^{-9}$ Torr housing the analyzer. The experimental data were calibrated with the carbon peak that was always shown as a contamination at $285 \mathrm{eV}$. The morphology of deposited gold was characterized by JEOL 6300F field emission scanning electron microscopy (FESEM) with secondary electron images at $30 \mathrm{kV}$, and with Digital Instruments 3100 atomic force microscopy (AFM) in contact mode.

An objective measure of the wavelength at which the plasmon resonance occurred was obtained by taking a numerical first derivative, $\frac{d T}{d \lambda}$ of the transmission intensity through the coating, versus wavelength. The maximum absorption was taken as occurring at the wavelength for which $\frac{d T}{d \lambda}=0$. The volume fraction of the gold in our coatings could not be properly determined by simple areal analysis, since the coatings were three dimensional at the nanoscale. Therefore, it was determined by constructing a geometric model of the surface using numerous measurements of particle diameter, position and shape made from the SEM and AFM images using custom-written software. The software allowed the user to map an image of the coating into an array of hemispheres. Since many 
of the particles were close together or overlapping, double-counting of gold was avoided by rendered the list of hemispherical particles into an array of voxels which represented a slab of thickness, $t$. The volume fraction, $\phi$, occupied by gold was then estimated from this array by point counting, and was influenced by the value chosen for $t$. Two measures of volume fraction were

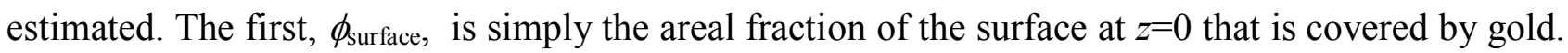
The second is the volume fraction, $\phi_{\text {box }}$, of gold in the slab bounded by $z=0$ and $z=t$, where $t$ is the maximum height of any particle in the relevant sample. Obviously $\phi_{\text {surface }}>\phi_{\text {true }}>\phi_{\text {box }}$, where $\phi_{\text {true }}$ is the actual volume fraction of gold a experienced by a photon. 


\section{Results}

Typical XPS spectra of $\mathrm{Au}(4 \mathrm{f})$ as-deposited onto the glass are shown in Fig. 2. for different processing times. $\mathrm{Au}\left(4 \mathrm{f}_{7 / 2}, 5 / 2\right)$ peaks at 84.0 and $87.9 \mathrm{eV}$ correspond to pure gold. With increasing immersion time in the coating solution, the area of the gold peaks increased (Fig. 3), while the area of the silicon $(2 s, 2 p)$ peaks reduced, which indicated an increase in the coverage of the gold nanoparticles on the glass substrate.

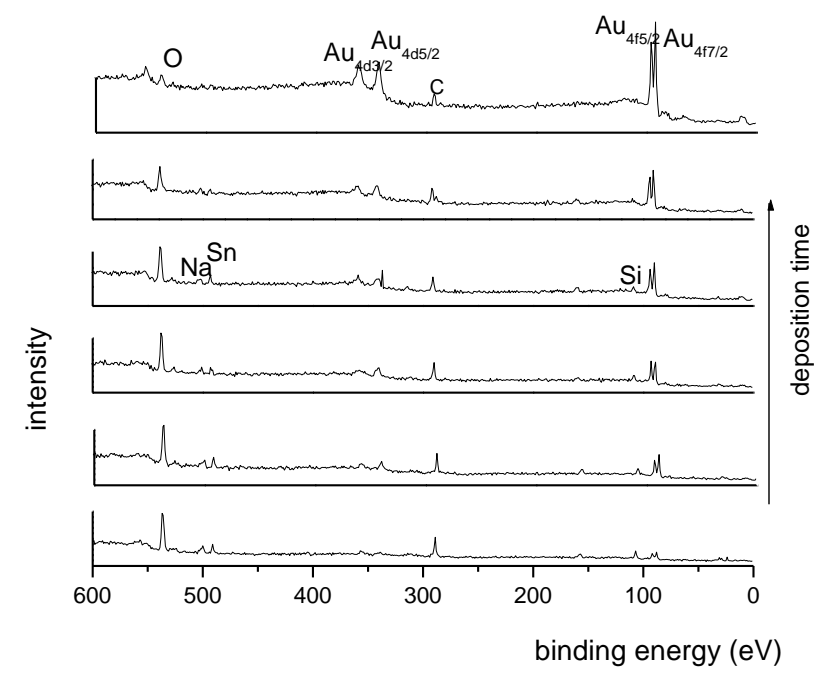

Fig. 2 XPS spectra of glass surfaces coated at $\mathrm{pH} 5.1$ for (from bottom to top) $6,12,18,24,30$ and 50 minutes respectively 


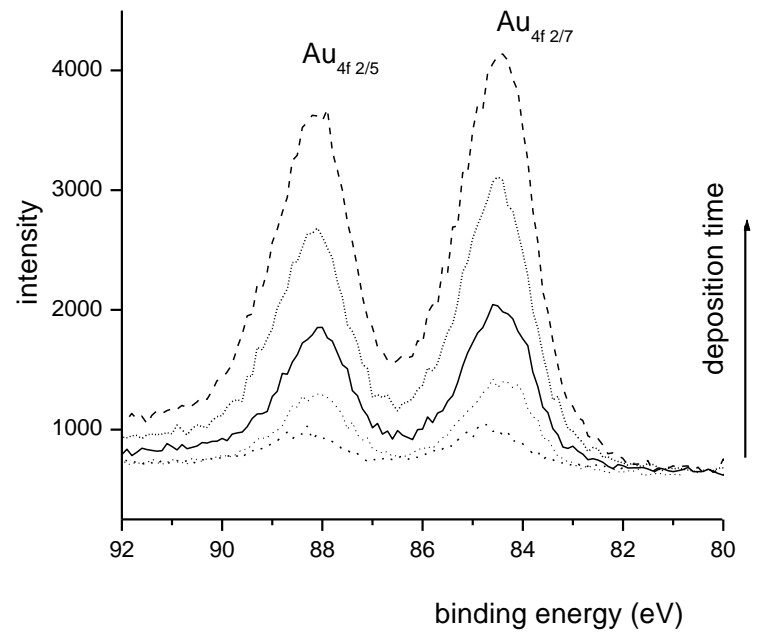

Fig. 3 Gold peaks on XPS spectra, showing development of coating of gold nanoparticles as a function of deposition time (deposited in $\mathrm{pH} 5.1$ solution for $6,12,30,40,60$ minutes respectively)

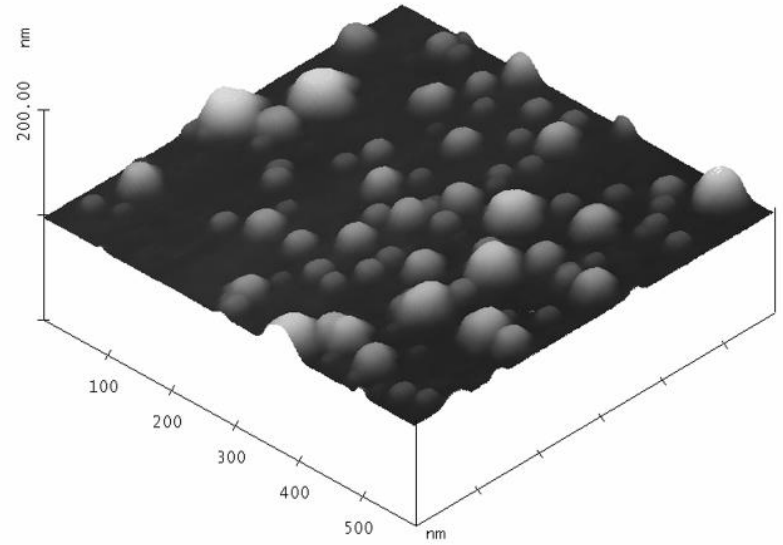

Fig. 4 AFM morphology of gold nano-particles deposited on glass 


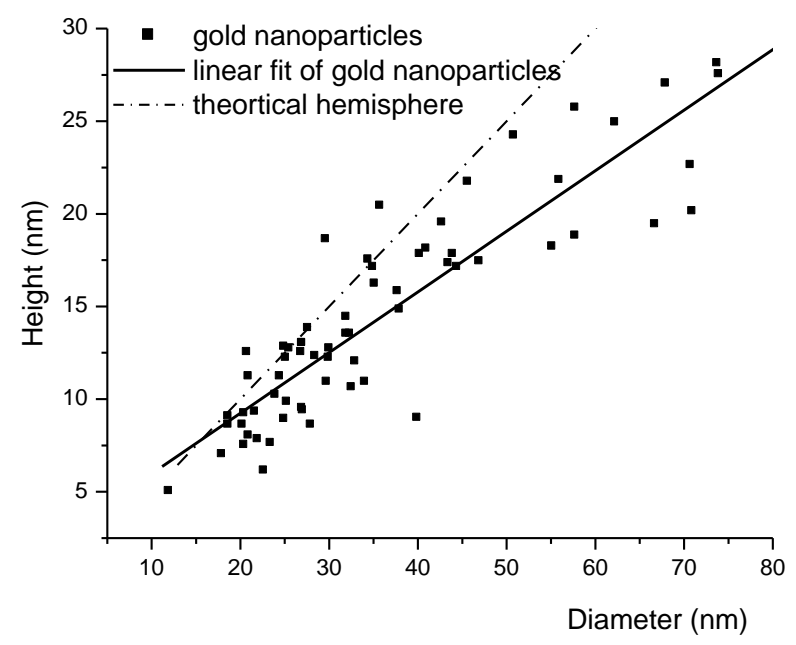

Fig. 5 Height and diameter of particles demonstrating hemispherical shape

An AFM image of the coating is shown in Fig. 4, while the measured aspect ratios of the particles in that image are plotted in Fig. 5. It is evident that the particles are approximately hemispheres, which indicates the reaction is in situ.

Fig. 6, 7 and 8 show the visible and infrared spectra of glass slides coated with gold at $\mathrm{pH} 5.1, \mathrm{pH}$ 8.0 and $\mathrm{pH} 10.0$ respectively. From these figures it is evident that the longer the deposition time, the lower the transmittance in both the visible and the infrared area. It is worth noting that the surface of the glass slides became coloured well-before any colloidally-induced colouring became visible in the coating solution. We interpret this as evidence that the nucleation and growth of the nanoparticles occurred first on the surface of the glass before it took place in the solution.

For the deposition at $\mathrm{pH} 5.1$, the spectrum can be grouped into three stages. In the initial stage, there is the appearance of the first absorbance peak at $520 \mathrm{~nm}$, which included spectrum I and II in Fig. 6. After prolonged immersion time a second peak appeared at $700 \mathrm{~nm}$ while the first peak was still visible (Figure 6 - III, IV and V). In this phase, the first peak is still at $520 \mathrm{~nm}$ while the second peak shifted gradually to $750 \mathrm{~nm}$ during the deposition. In the last stage, the second peak broadened 
and shifted further to longer wavelengths, and the first peak was no longer discernible on the spectrum. Meanwhile, the transmittance of glass slide in $2500 \mathrm{~nm}$ (indicated infrared region) rapidly dropped from $78 \%$ to $7 \%$ (Fig. 6 - VI, VII, VIII and IX). In these three stages, the colour of the glass slide varied in hue from pink through violet to blue.

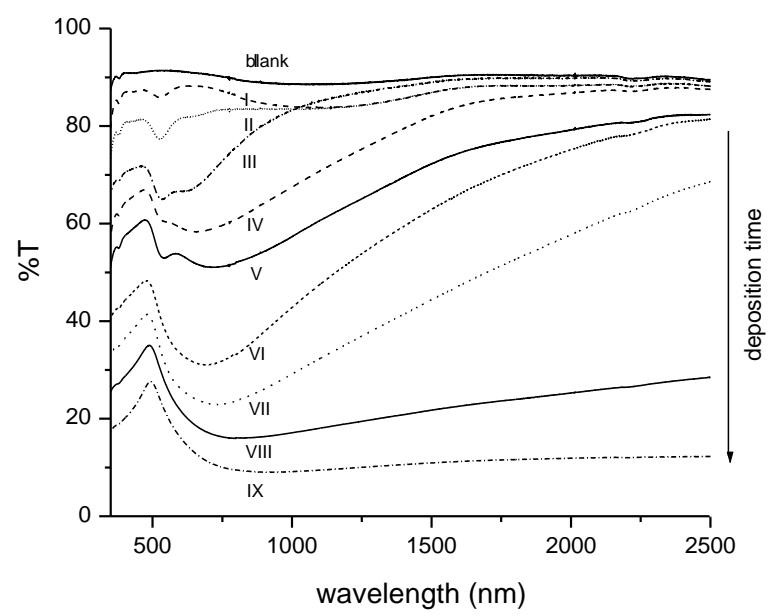

Fig. 6 Transmittance spectra of gold nanoparticles deposited at a $\mathrm{pH}$ of 5.1, shown for deposition times of $6,12,18,24,30,36,40,50,60$ minutes, corresponding to spectra I through IX respectively.

For the deposition at $\mathrm{pH} 8.0$, the spectra can be grouped into two types. The first absorbance peak appeared at $520 \mathrm{~nm}$ (Fig. 7 I). This peak broadened quickly during the deposition process (Fig. 7 II). The second peak appeared at $720 \mathrm{~nm}$ and moved further to the infrared area during the deposition process (Fig. 7 III- VIII). The transmittance of the glass in near infrared area dropped from $85 \%$ to $10 \%$. 


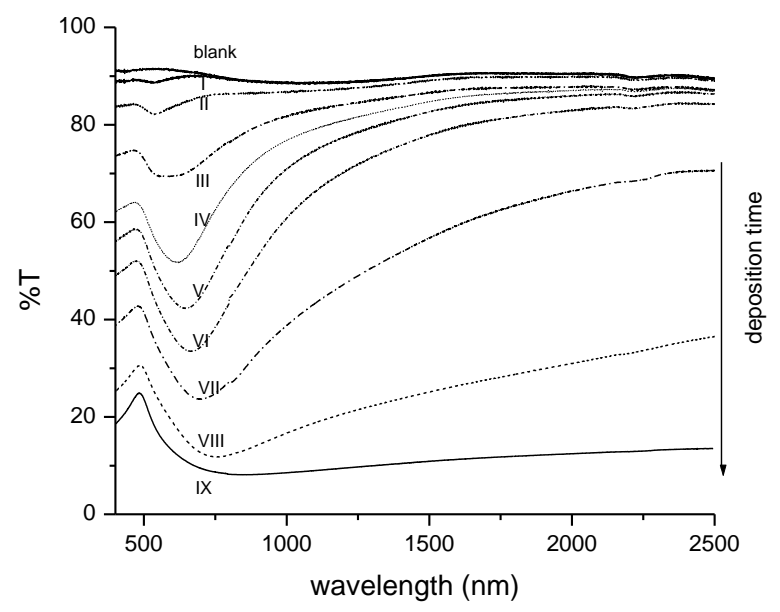

Fig. 7 Transmittance spectra of gold nanoparticles deposited at a $\mathrm{pH}$ of 8.0, shown for deposition times of $2,6,12,18,24,30,42,45$ and 50 minutes, corresponding to spectra I through IX respectively

For the deposition at $\mathrm{pH} 10.0$, the spectra are quite similar to those of $\mathrm{pH} 8.0$. There was no clear coexistence of the first and second peaks on the spectra. The first absorbance peak appeared at 520 nm (Fig. 8 I), but was quickly overwhelmed by the development of the second peak during the deposition process. The second peak appeared at $720 \mathrm{~nm}$ and moved further to the infrared area as the coating thickened (Fig. 8 II- VIII). The transmittance of the glass in near infrared area dropped from $85 \%$ to $10 \%$. The colour of the glass slide varied in hue from pink to blue directly. It seems that there was no obvious transition through violet in both $\mathrm{pH} 8.0$ and 10.0. 


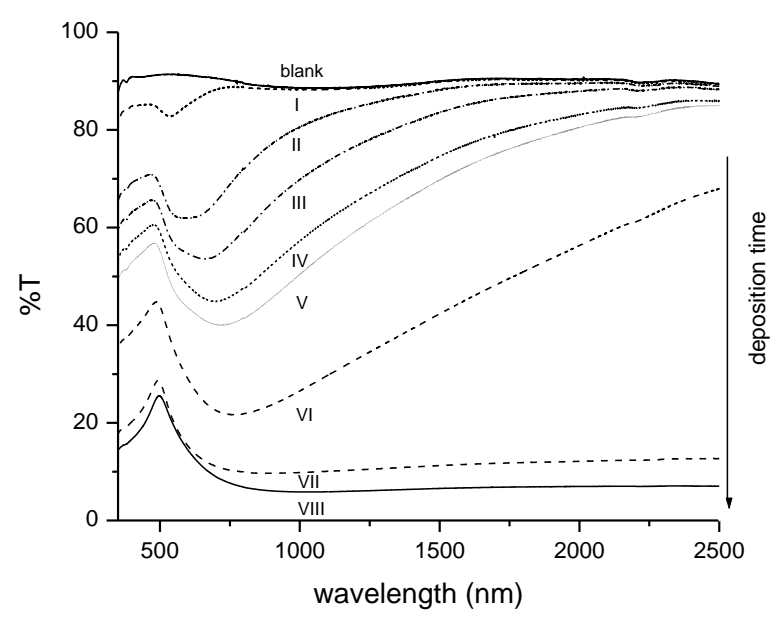

Fig. 8 Transmittance spectra of gold nanoparticles deposited at a $\mathrm{pH}$ of 10.0, shown for deposition times of $2,6,12,18,24,30,35$ and 40 minutes, corresponding to spectra I through VIII respectively

Fig. 9 shows the morphology of gold nano-particles deposited at different process phases at a $\mathrm{pH}$ of 5.1. There is an increase of the coverage of the substrate, in agreement with the XPS results. The typical morphology of nanoparticles of first stage is displayed in Fig. 9 a, b. It was shown that the nanoparticles grew from $25 \mathrm{~nm}$ (Fig. 9 a) to $50 \mathrm{~nm}$ (Fig. 9 b). Most of the gold nano-particles at this stage were isolated. Fig. $9 \mathrm{c}$ is the typical morphology of nanoparticles in the second stage. In this stage, many clusters formed in between isolated nanoparticles. The size of gold nanoparticles stayed at around $50 \mathrm{~nm}$ however. After that, the substrate was covered by the nanoparticles and clusters completely (Fig. 9 d), and aggregated gold nano-particles merged into large irregular shapes of nanoscale dimensions, to form a continuous thin film. The shape of the original gold nanoparticles can still be discerned after this agglomeration.

In Fig. 10 we show the morphology of gold nano-particles deposited at different phases of the process at $\mathrm{pH}$ 10.0. In the first stage (Fig. 10 a) it is seen that the size of nano-particles was similar to that deposited at $\mathrm{pH} 5.1$, which is around $25 \mathrm{~nm}$. However, the density of nanoparticles is quite 
high compared with that deposited at $\mathrm{pH}$ 5.1. In the second stage (Fig. $10 \mathrm{~b}, \mathrm{c}, \mathrm{d}$ ), clusters of aggregated gold nano-particles were formed. The substrate was completely covered by these gold clusters. The size of gold nanoparticles stayed at around $30 \mathrm{~nm}$ (Fig. $10 \mathrm{~b}$ ), which is significantly smaller than for the gold deposited at $\mathrm{pH}$ 5.1. Finally, the clusters merged into a continuous film. Once again, the shape of the original nanoparticles can still be observed (Fig. 10 c, d). 

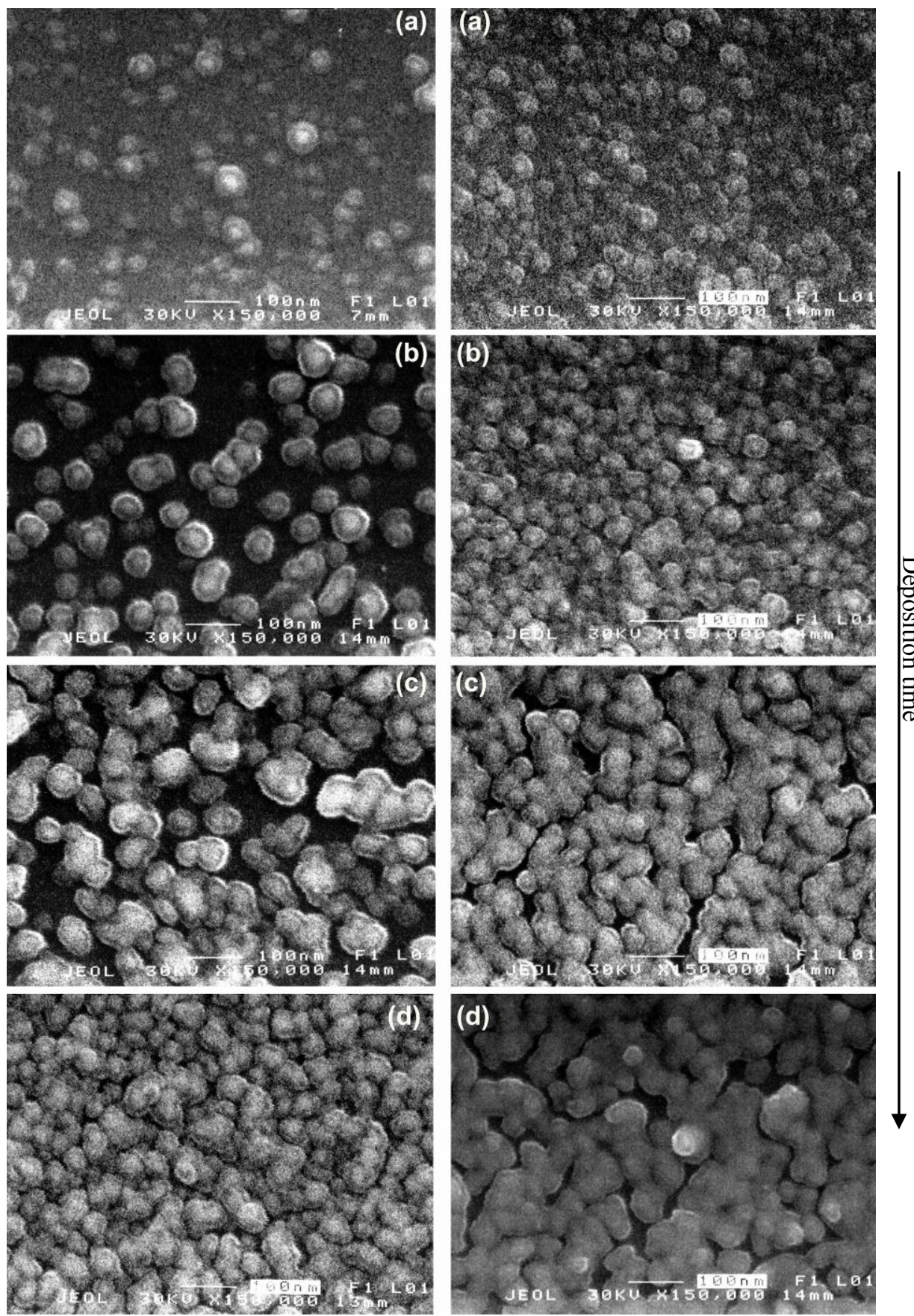

Fig. 9 Morphology of gold nano-

Fig. 10 Morphology of gold particles deposited at $\mathrm{pH} 5.1$

nanoparticles deposited at $\mathrm{pH} 10.0$ 


\section{Discussion}

\section{Color as a function of volume fraction of gold}

The change of color of the coatings is due to the surface plasmon polariton, which is an electromagnetic mode existing at the interface between metal and dielectric. Electromagnetic radiation can penetrate 20 to $30 \mathrm{~nm}$ into gold at visible wavelengths, which is comparable to the size of the nanoparticles themselves. In this case the electromagnetic wave forces oscillations of the free electrons in the particle with the same frequency as the applied electric field. A plasmon resonance will occur at a frequency depending on material and geometry. The absorption peak for isolated gold nanoparticles in the 3 to $40 \mathrm{~nm}$ size range is at about $520 \mathrm{~nm}$. ${ }^{11}$ The first peak in both series of our experimental spectra is therefore obviously caused by such particles on the glass surface.

The second peak at around $700 \mathrm{~nm}$ can be explained by dipole-dipole model interactions between nearby nanoparticles. ${ }^{12,26}$ The optical absorption of the nanoparticles due to this effect is proportional to $(R / d)^{2 \mathrm{~L}+1}$, where $R$ is particle radius, $d$ is the interparticle distance, and $L$ is the multipole order ( $L=1$ for dipole, $L=2$ for quadrupole, etc). ${ }^{26}$ It is obvious that reducing the interparticle distance and/or increasing the particle diameter will increase this source of absorption. The presence of this peak is therefore indicative of aggregation of the particles e.g. Natan. ${ }^{17}$

Genzel and Martin have shown that an approximate expression to model these effects may be obtained by noting that the plasmon resonance of non-contiguous nanoparticles will occur when ${ }^{27}$

$$
\varepsilon_{A u}^{\prime}(\lambda)=-\varepsilon_{m}^{\prime}(\lambda) \frac{(2+\phi)}{(1-\phi)}
$$

where $\lambda$ is the wavelength of the light, $\varepsilon_{A u}^{\prime}(\lambda)$ is the real part of the dielectric constant of gold, $\varepsilon_{m}^{\prime}(\lambda)$ is the real part of the effective dielectric constant of the matrix surrounding the gold particle, 
and $\phi$ is the volume fraction of gold particles. Both $\varepsilon_{A u}^{\prime}(\lambda)$ and $\varepsilon_{m}^{\prime}(\lambda)$ may be functions of $\lambda$, but $\varepsilon_{m}^{\prime}(\lambda)$ is often taken as a constant. ${ }^{8,27}$ Experimental values for $\varepsilon_{A u}^{\prime}(\lambda)$ may be found in the literature, and here we have fitted published data, ${ }^{28}$ with a fourth order polynomial to yield an empirical

$$
\varepsilon_{A u}^{\prime}(\lambda) \approx \sum_{i=0}^{4} a_{i} \lambda^{i}
$$

where $a_{0}=3.3777 \mathrm{E}+01, a_{1}=-3.6141 \mathrm{E}-01, a_{2}=1.3083 \mathrm{E}-03, a_{3}=-1.9057 \mathrm{E}-06$ and $a_{4}=8.8682 \mathrm{E}-10$. The particle size does not come explicitly into the Genzel-Martin expression. However, a more detailed analysis of the condition for plasmon resonance includes contributions from particle size, ${ }^{11}$ and it is these factors that explain the small red-shifting with increase in size that is also observed in practice.

The matrix around the particles consists of soda lime glass on one side, with $\varepsilon$ of about 7 , and air on the other with $\varepsilon$ of 1 . Since, unlike for metals, the values of $\varepsilon$ for air and glass are not particularly sensitive to wavelength, we will replace $\varepsilon_{m}^{\prime}(\lambda)$ in Equation (1) with a single value that is an effective average of the two media. The observed position of the plasmon resonance at the start of the experiments, for which $\phi<<1$, is $520 \mathrm{~nm}$. Substitution of this value, $\phi=0$, and the data of Equation (2) into Equation (1), indicates a value for $\varepsilon_{m}^{\prime}$ of approximately 1.75.

The position of the plasmon resonance due to dipole-dipole interactions can now be estimated as a function of $\phi$. Of course this expression is strictly speaking correct only for discrete, mono-disperse gold nano-particles. Furthermore, it ignores not only multipole interactions that will become very important as $\phi$ increases above about $0.5,{ }^{29}$ but also that the shape of the particles plays a role, and that the dipole-dipole interaction depends actually on interparticle distance, not on volume fraction. This latter point has been elegantly demonstrated by Rechberger et al. for lithographically prepared arrays of identical average volume fraction, and we will return to it later. ${ }^{30}$ 
The data of Ung et al, ${ }^{13}$ derived for very precisely determined values of $\phi$, are shown together with Equation (1) in Fig. 11. The agreement is excellent, notwithstanding any objections raised regarding the excessive simplicity of Equation (1). Therefore, we believe that there is some utility in examining how well it explains the present data. Two trends, corresponding to the upper (areal) and lower (box) estimates of volume fraction described earlier are shown.

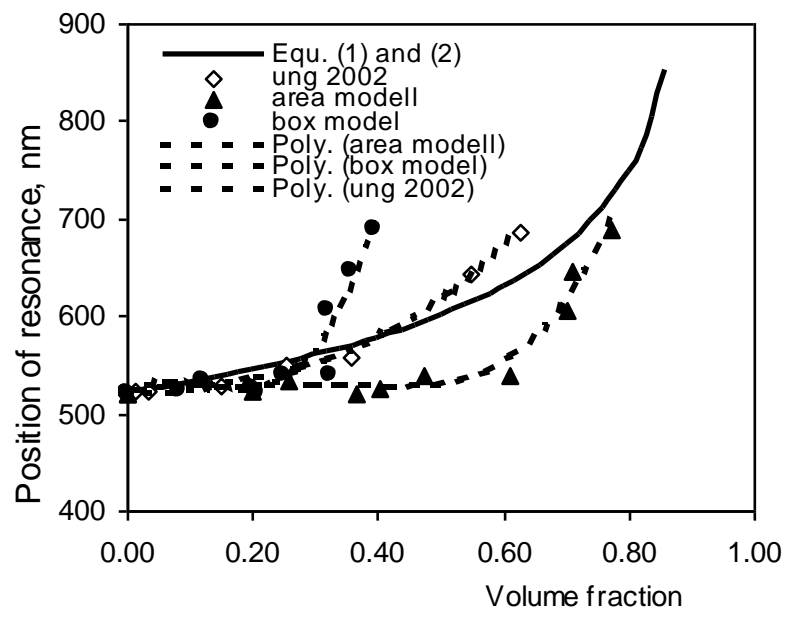

Fig. 11 Effect of volume fraction on position of resonance peak

It is evident that, as expected, the areal estimate overestimates the volume fraction, while the box one underestimates it, and that these two alternatives straddle the predictions of Equation (1). In addition, the wavelength of maximum adsorption increases somewhat faster, at higher volume fractions, than expected. This is due to the dipole interactions mentioned earlier. The fit can be improved by re-addressing the nature of the 'volume fraction' calculated but we will not pursue this point here.

The smooth variation of hue from pink through violet to blue of the coatings deposited at $\mathrm{pH} 5.1$ can therefore be explained as follows. At first the density of particles was low, so that most of the particles are isolated. This produces the well-known absorption peak at $520 \mathrm{~nm}$ without other peaks. In the second stage, while the density is still relatively low, two factors developed concurrently. 
First, the isolated particles grew and second, some of particles aggregated as clusters. In this stage, two peaks (see Fig. 6 III, - V) coexisted, with the new peak at $700 \mathrm{~nm}$ due to the resonance of the aggregated nanoparticles, while the original plasmon resonance at $520 \mathrm{~nm}$ has become somewhat red-shifted. In the third stage, there were few isolated nanoparticles left on the surface and the first plasmon resonance peak is no longer visible in the spectrum due to the significant broadening of the new peak. The $700 \mathrm{~nm}$ peak had also red-shifted due to the reduction of distance between dipoles caused by further agglomeration of the clusters.

At $\mathrm{pH} 10.0$, the deposition rate was so fast that even at the first stage the density of the nanoparticles was very high. There was not enough space for growth of isolated particles. Rather a large number of agglomerated particles formed from early on during the deposition. These factors also restricted the growth of the nanoparticles themselves. This explains why the peaks at $520 \mathrm{~nm}$ and $700 \mathrm{~nm}$ do not co-exist in these samples beyond the very earliest stages. As with the samples at $\mathrm{pH} 5.1$, the decrease in interparticle distance, led to red-shifting and broadening of the second peak.

The optical properties of gold nanoparticles on the glass substrate are therefore mainly determined by two factors: one is the property of the gold nanoparticles acting as well-isolated individuals; another is the collective properties of clusters. Both contributions must be taken into consideration. The influence of the second factor is more pronounced at high particle densities.

\section{Color as a function of particle morphology}

As mentioned, the color depends primarily of interparticle separation, and on particle morphology, and not actually on volume fraction. A demonstration of this effect was also found in the present work, during the course of which it became evident that even in coatings of similar average density and similar nominal particle size, different patterns of aggregation could result in quite different spectra. In Fig. 12, we show two coatings of similar density and particle size, but different styles of 
aggregation. Fig. 12 (a) shows substantially isolated particles of $60 \mathrm{~nm}$ diameter, and the corresponding spectrum shows a peak at $520 \mathrm{~nm}$, as expected from individual particles. In Fig. 12 (b) we see nano-particle clusters that are aggregated from much smaller particles. The corresponding spectrum was characterised by a broad absorption at $\sim 655 \mathrm{~nm}$ due to dipole-dipole interactions between the aggregated nanoparticles, and the peak at $520 \mathrm{~nm}$ has been swamped in the spectrum. However, the areal and 'box' volume fractions of the two samples are not statistically different, Table 1. The importance of this observation is that it shows how blue-shifted films can actually be obtained without necessarily overtly sacrificing transparency of the coating. This is useful in the context of architectural applications.

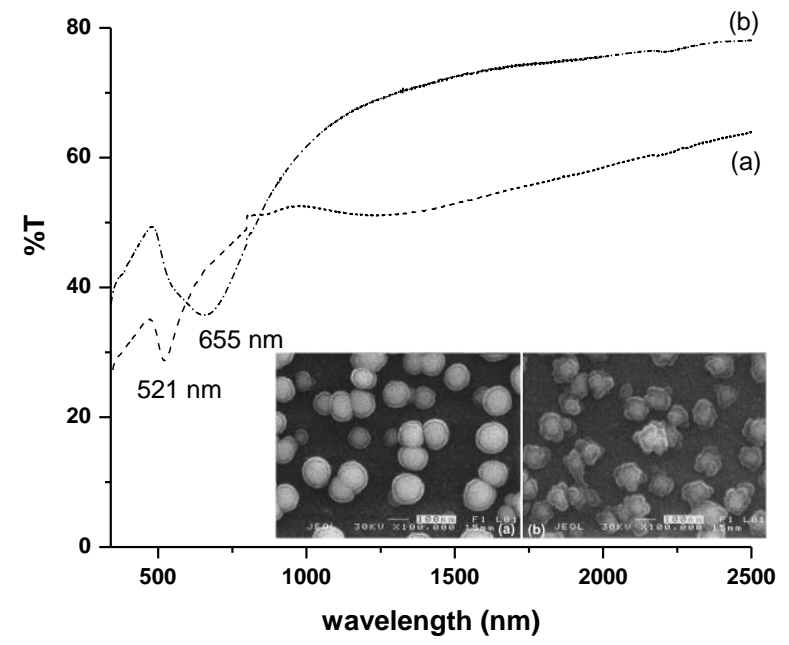

Fig. 12 Influence of different patterns of clustering on plasmon resonance peak position

\section{Conclusions}

This study investigated the effect of $\mathrm{pH}$ on the optical properties of gold nano-particle coatings grown on glass. The work has been motivated by the desire to develop window coatings that would attenuate solar radiation while exhibiting an acceptable hue. The particles appeared to nucleate and 
grow directly on the surface of the glass, rather than in the coating solution. This was deduced from their hemispherical morphology. Coatings comprised of isolated gold nanoparticles exhibited the well-known plasmon resonance at $\sim 520 \mathrm{~nm}$ on the glass substrate and were pink-coloured. However, the absorption peak due to particle-particle interactions, which is at about $700 \mathrm{~nm}$, could be developed with increase of coating density. This peak could be broadened and red-shifted by increasing the deposition time. Such coatings were blue or blue-grey in transmission, and would be more suitable in principle for architectural applications than the pink ones. The most desirable bluegrey coatings were obtained after deposition at $\mathrm{pH} 10$, and displayed a significantly different morphology than those deposited at a $\mathrm{pH}$ of 5.1. The extent to which the position of the plasmon resonance can be modelled as a function of volume fraction was assessed, and the reasons for deviations from this model examined. 


\section{Acknowledgements}

The authors thank Professor G.B. Smith, Dr R. Wuhrer, Mr. G. McCredie, Dr M. Zareie and Mr S. Schelm of the University of Technology Sydney for assistance received in the course of this work. 
Table 1 Influence of aggregating mode on resonance plasmon peak. Number of measurements, $n$, and standard deviation, $s$, in brackets.

\begin{tabular}{|c|c|c|c|c|}
\hline Sample & $\begin{array}{l}\text { Areal } \\
\text { fraction }\end{array}$ & $\begin{array}{l}\text { 'Box' } \\
\text { volume } \\
\text { fraction }\end{array}$ & $\begin{array}{l}\text { Average radius } \\
\text { of hemisphere } \\
\text { in model, nm }\end{array}$ & $\begin{array}{l}\text { Plasmon } \\
\text { resonance, } \\
\mathrm{nm}\end{array}$ \\
\hline $\begin{array}{ll}\text { (a) } & \text { Single } \\
\text { isolated } \\
\text { round }\end{array}$ & $\begin{array}{l}0.360 \\
(9,0.102)\end{array}$ & $\begin{array}{l}0.175 \\
(9,0.045)\end{array}$ & $57.8(956,16.7)$ & 521 \\
\hline $\begin{array}{l}\text { (b) Aggregated } \\
\text { small } \\
\text { particles }\end{array}$ & $\begin{array}{l}0.375 \\
(6,0.032)\end{array}$ & $\begin{array}{l}0.167 \\
(6,0.021)\end{array}$ & $21.4(1448,8.0)$ & 655 \\
\hline
\end{tabular}




\section{Index of Figures}

Fig. 1 Direct normal solar spectral irradiance at air mass 1.5 for a $37^{\circ}$ tilted surface ${ }^{2}$

Fig. 2 XPS spectra of glass surfaces coated at pH 5.1 for (from bottom to top) 6, 12, 18, 24, 30 and 50 minutes respectively

Fig. 3 Gold peaks on XPS spectra, showing development of coating of gold nanoparticles as a function of deposition time (deposited in $\mathrm{pH} 5.1$ solution for $6,12,30,40,60$ minutes respectively)

Fig. 4 AFM morphology of gold nano-particles deposited on glass

Fig. 5 Height and diameter of particles demonstrating hemispherical shape

Fig. 6 Transmittance spectra of gold nanoparticles deposited at a $\mathrm{pH}$ of 5.1, shown for deposition times of $6,12,18,24,30,36,40,50,60$ minutes, corresponding to spectra I through IX respectively.

Fig. 7 Transmittance spectra of gold nanoparticles deposited at a $\mathrm{pH}$ of 8.0, shown for deposition times of $2,6,12,18,24,30,42,45$ and 50 minutes, corresponding to spectra I through IX respectively

Fig. 8 Transmittance spectra of gold nanoparticles deposited at a $\mathrm{pH}$ of 10.0, shown for deposition times of $2,6,12,18,24,30,35$ and 40 minutes, corresponding to spectra I through VIII respectively

Fig. 9 Morphology of gold nano-particles deposited at pH 5.1

Fig. 10 Morphology of gold nanoparticles deposited at $\mathrm{pH} 10.0$ 
Fig. 11 Effect of volume fraction on position of resonance peak

Fig. 12 Influence of different patterns of clustering on plasmon resonance peak position 


\section{References}

(1) Johnson, T.E. Low-E Glazing Design Guide; Butterworth Architecture: Boston, 1991.

(2) ASTM G 159 - 98. Standard Tables for References Solar Spectral Irradiance at Air Mass 1.5: Direct Normal and Hemispherical for a $37^{\circ}$ Tilted Surface; American Society For Testing And Materials: West Conshohocken, PA, 1998.

(3) Wruk, N. Glass Processing Days 1997. 13-15 ${ }^{\text {th }}$ September 1997, Tampere, Finland, 182.

(4) Block, V. Glass Processing Days 2001, $18^{\text {th }}-21^{\text {st }}$ June 2001, Tampere, Finland, 826.

(5) Bell, J.M. ; Matthews, J.P. Materials Forum, 1998, 22, 1.

(6) Nadel, S.J. ; Hill, R.J. Glass Processing Days 1997, 13-15 ${ }^{\text {th }}$ September 1997, Tampere, Finland, 209.

(7) Kühnel, F. ; Paul, T. Glass Processing Days 2001, $18^{\text {th }}-21^{\text {st }}$ June 2001, Tampere, Finland, 760 .

(8) Smith, G.B. ; Deller, C.A. ; Swift, P.D. ; Gentle, A. ; Garrett, P.D. ; Fisher, W.K. J. Nanoparticle Research. 2002, 4, 157.

(9) Takeda, H. ; Yabuki, K. ; Adachi, K. United States Patent 6,319, 613, 2001.

(10) Genzel, L. ; Martin, T.P. Z. Physik B. 1975, 21, 339. 
(11) Bohren, C.F. ; Huffman, D.R. Absorption and Scattering of Light by Small Particles; Wiley-Interscience: New York, 1998.

(12) Turkevich, J. Gold Bulletin. 1985, 18, 125.

(13) Ung, T. ; Liz-Marzán, L.M. ; Mulvaney, P. Colloids and Surfaces A: Physicochemical and Engineering Aspects 2002, 202, 119.

(14) Félidj, N. ; Aubard, J. ; Lévi, G. ; Krenn, J.R. ; Schider, G. ; Leitner, A. ; Aussenegg, F.R. Physical Review B 2002, 66, 245407.

(15) Mulvaney, P. Langmuir, 1996, 12, 788.

(16) Seitz, O. ; Chehimi, M.M. ; Cabet-Deliry, E. ; Truong, S. ; Felidj, N. ; Perruchot, C. ; Greaves, S.J. ; Watts, J.F. Colloids and Surfaces A: Physicochemical and Engineering Aspects 2003, 218, 225.

(17) Natan, M.J. ; Baker, B.E. United States Patent 6,242,264, 2001.

(18) Park, S.H. ; Im, J.H. ; Im, J.W. ; Chun, B.H. ; Kim, J.H. Microchemical Journal 1999, 63, 71.

(19) Miller, R.G. ; Cavitt, R.L. United States Patent 4,005,229, 1977.

(20) Westcott, S.L. ; Oldenburg, S.J. ; Randall Lee, T. ; Halas, N.J. Langmuir, 1998 14, 5396.

(21) Levy, D.J. United States Patent 3,515,571, 1970.

(22) Franz, H. ; Vanek, J.C. United States Patent 4,091,128, 1978. 
(23) Luce, B.M. United States Patent 3,300,328, 1967.

(24) Breitinger, D.K. ; Herrmann, W.A. Synthetic Methods of Organometallic and Inorganic Chemistry, Vol.5. George Thieme Verlag: Stuttgart, New York, 1999.

(25) Schmidbauer, H. Gold: Progress in Chemistry, Biochemistry and Technology. John Wiley and Sons: Chichester, 1999.

(26) Liu, Z.; Wang, H. ; Li, H. Applied Physics Letters 1998, 15, 1823.

(27) Genzel, L. ; Martin, T.P. Surface Science, 1973, 34, 33.

(28) Johnson, P.B. ; Christy, R.W. Physical Review 1972, B6, 4370.

(29) Smith, G.B. University of Technology Sydney, Private communication, July 2003.

(30) Rechberger, W. ; Hohenau, A. ; Leitner, A. ; Krenn, J.R. ; Lamprecht, B. ; Aussenegg, F.R. Optics Communications 2003, 220, 137. 